\title{
New Atomic Force Microscope Facilitates Faster Workflow for Nanoscale In Situ Applications
}

\author{
Christian Rankl, ${ }^{\star}$ Gerald Kada, Shijie Wu, and Anil Ghimire
}

Keysight Technologies, 5301 Stevens Creek Blvd, Santa Clara, CA 95051

*christian_rankl@keysight.com

Abstract: The latest generation of atomic force microscopes (AFMs) adds ease-of-use and quick imaging to the long list of established capabilities: high spatial resolution, advanced sample characterization, and high-force resolution. This allows even the novice user to quickly capture dynamic processes with high resolution. Fast image acquisition has been used here to investigate the phase transition of a polymer and to observe the formation of ice crystals in real time with nanometer resolution. Fast acquisition does not compromise other capabilities like electrical characterization. Kelvin-force microscopy was used for a quantitative investigation of the surface potential of a fluoroalkane at the sub-molecular level.

\section{Introduction}

A growing number of scientists and engineers around the world are using atomic force microscopy (AFM) [1] for an ever more diversified range of academic and industrial applications. Now three decades old, this popular nano-measurement technology is experiencing many advancements as users working in many fields seek AFM instrumentation that delivers data faster, affords more comprehensive experimental control, and is simpler to operate.

The principle of AFM imaging is based on moving a sharp tip across a sample surface in a raster motion, while, in the case of contact mode imaging, the deflection is kept constant by retracting and approaching the tip using a feedback loop. Therefore, the tip is following the surface topography, and sample images are generated from this tip motion. Optimizing those feedback parameters is a major barrier for AFM beginners. Up to now it required considerable experience to get good images with high resolution and without artifacts.

A major advantage of the AFM is that it provides images with sub-nm spatial resolution [2], orders of magnitude better resolution than with diffraction limited light optical microscopy ( 200 nm). Furthermore, it can measure forces with pico-Newton resolution. In fact, it is used in biology to investigate single-molecule interactions [3]. A major disadvantage of this class of instruments is that image acquisition is slow ( $\sim 5 \mathrm{~min})$. This limits its throughput and its applicability for capturing dynamic processes.

The recently introduced 9500 AFM (Keysight Technologies Inc, [4]) addresses each of those points. This system has a state-ofthe-art mechanical design, with a low-latency, high-bandwidth digital controller. The software was redesigned to be intuitive, and the system has been optimized to streamline workflows. Furthermore, the newly developed AutoDrive technology automatically sets up the feedback loop parameters. The QuickScan option allows the user to get images within seconds instead of minutes.

In this article we describe the capture of dynamic processes with this new system. In particular, we investigate the phase transition, from partially crystalline to the amorphous state, for poly(diethylsiloxane) (PDES), as well as, the formation of ice crystals. These examples show how this instrument could be used for in situ experiments in the fields of materials science, life science, polymer science, and electrical characterization.

\section{Materials and Methods}

AFM imaging. All AFM imaging was done using a Keysight Technologies Inc. (Santa Rosa, CA) 9500AFM system with a $90 \mu \mathrm{m}$ closed-loop scanner with the QuickScan option. The probes used were Arrow UHF cantilevers from NanoWorld (Neuchâtel, Switzerland). The AFM was controlled by NanoNavigator, the new imaging and analysis software for the 9500AFM. Imaging was done in closed-loop mode to have best drift stability and linearity. The imaging parameters were automatically set by NanoNavigator using AutoDrive.

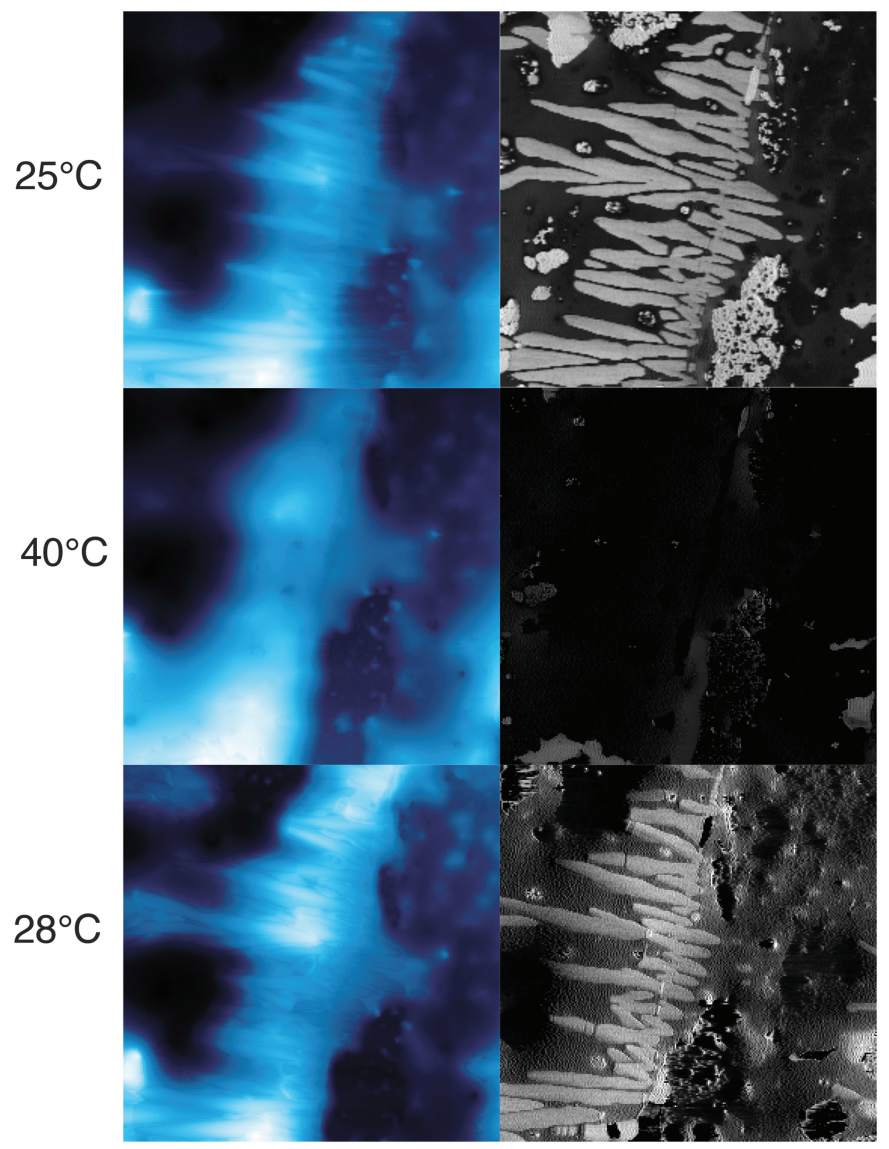

Figure 1: Investigation of PDES melting and recrystallization using quick AFM imaging. AFM imaging at 0.1 frames/s of a PDES sample was performed during a heating and cooling cycle. Scan size $=4 \mu \mathrm{m} \times 4 \mu \mathrm{m}$. The left images show the sample topography, while the right images show the simultaneously recorded phase images. It can be seen that during heating the crystals disappear in the topography and phase image. Because this process is reversible the crystals form again during cooling. 
The C-flat ${ }^{\text {Tm }}$ Advantage

C-flat ${ }^{\text {TM }}$ leads to

better data sets.

Made with patented technology, C-flat ${ }^{\mathrm{TM}}$ provides an ultra-flat surface that results in better particle dispersion and more uniform ice thickness.

Patterning is done using deep-UV projection lithography, ensuring the most accurate and consistent hole shapes and sizes down to submicron features. The precise methods by which C-flat ${ }^{\mathrm{TM}}$ is manufactured eliminate artifacts such as excess carbon and edges around holes.

\section{C-flat ${ }^{\mathrm{TM}}$ is affordable}

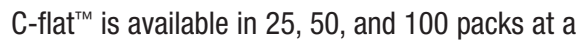
per-grid price less than competing products.

\section{Applications}

C-flat holey carbon grids provide the ideal specimen support to achieve high resolution data in cryoTEM making C-flat the perfect choice for:

- Single particle analysis

- Cryo electron tomography

- Automated TEM analysis

\section{mins has ith}

CONTACT US FOR MORE INFORMATION...

\section{Electron}

Microscopy Sciences

\author{
P.0. Box $550 \bullet 1560$ Industry Rd. \\ Hatfield, Pa 19440 \\ Tel: (215) 412-8400 • Fax: (215) 412-8450 \\ email: sgkcck@aol.com \\ or stacie@ems-secure.com
}

OUR MAIN INTERACTIVE WEBSITE:

\section{Www.emsdiasum.com}

\section{TO REQUEST A COPY}

OF OUR CATALOG: WwW.emsdiasum.com/ requests/catalog

\section{TO VIEW OUR}

DIGITAL CATALOG:

catalog.emsdiasum.com

the premier holey carbon grid for

cryo-transmission electron microscopy

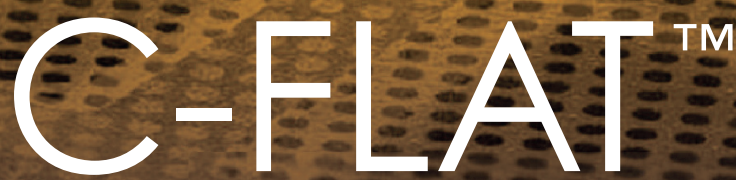

\section{Holey Carbon and Gold Grids for Gryo-TEM}

C-Flat ${ }^{T M}$ is a clean, ultra-flat holey carbon film TEM grid primarily used for Cryo TEM and Automated TEM. With a variety of available hole diameters, mesh size, film thicknesses, and mesh material, there is a C-Flat ${ }^{\text {TM }}$ product suitable for any application in the TEM.

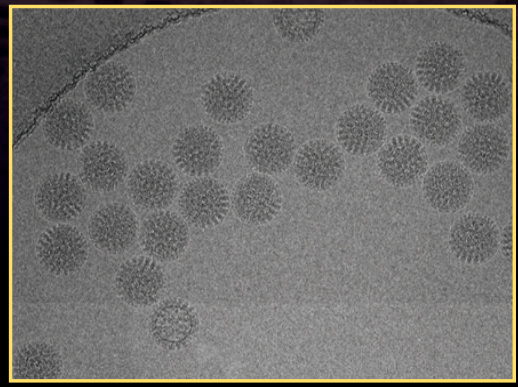

Frozen-hydrated Bacteriophage Capsid (data acquired on CF-1.2/1.4-4C).
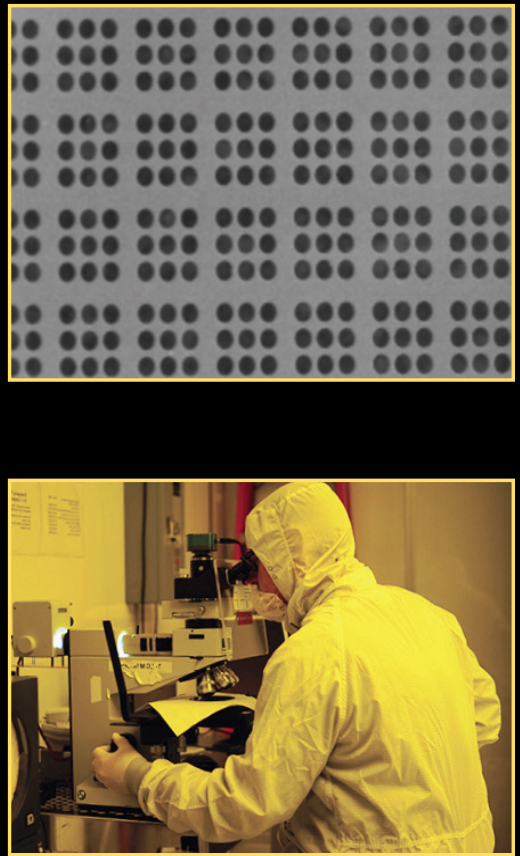

\section{Expanded Product Line}

The breadth of applications in cryoTEM necessitate a wide range of holey carbon film patterns. And now, with the recent expansion of the product line, a C-flat ${ }^{\mathrm{TM}}$ holey carbon film is available for almost any application.

\section{Consistent}

Researchers around the world have reported that the ultra-flat surface of $\mathrm{C}$-flat ${ }^{\text {TM }}$ leads to even ice thickness and uniform particle distribution, allowing for superior 3-D reconstructions. $2 \mu \mathrm{m}$ hole sizes are standard, but various hole sizes are available to accommodate different particle sizes and magnifications.

\section{Compatible}

C-flat ${ }^{\text {TM }}$ provides a regular array of analysis sites compatible with automated data collection software such as Leginon. This compatibility, in combination with the more uniform ice thickness and particle distribution reported by numerous researchers, results in more high-quality target sites per grid.

\section{Clean}

C-Flat ${ }^{\text {TM }}$ uses no plastics or polymers in its production. This means C-Flat ${ }^{\mathrm{TM}}$ is shipped clean, so it's ready to use out of the box and requires no solvent washing steps prior to use, leading to less breakage of the holey carbon film. 


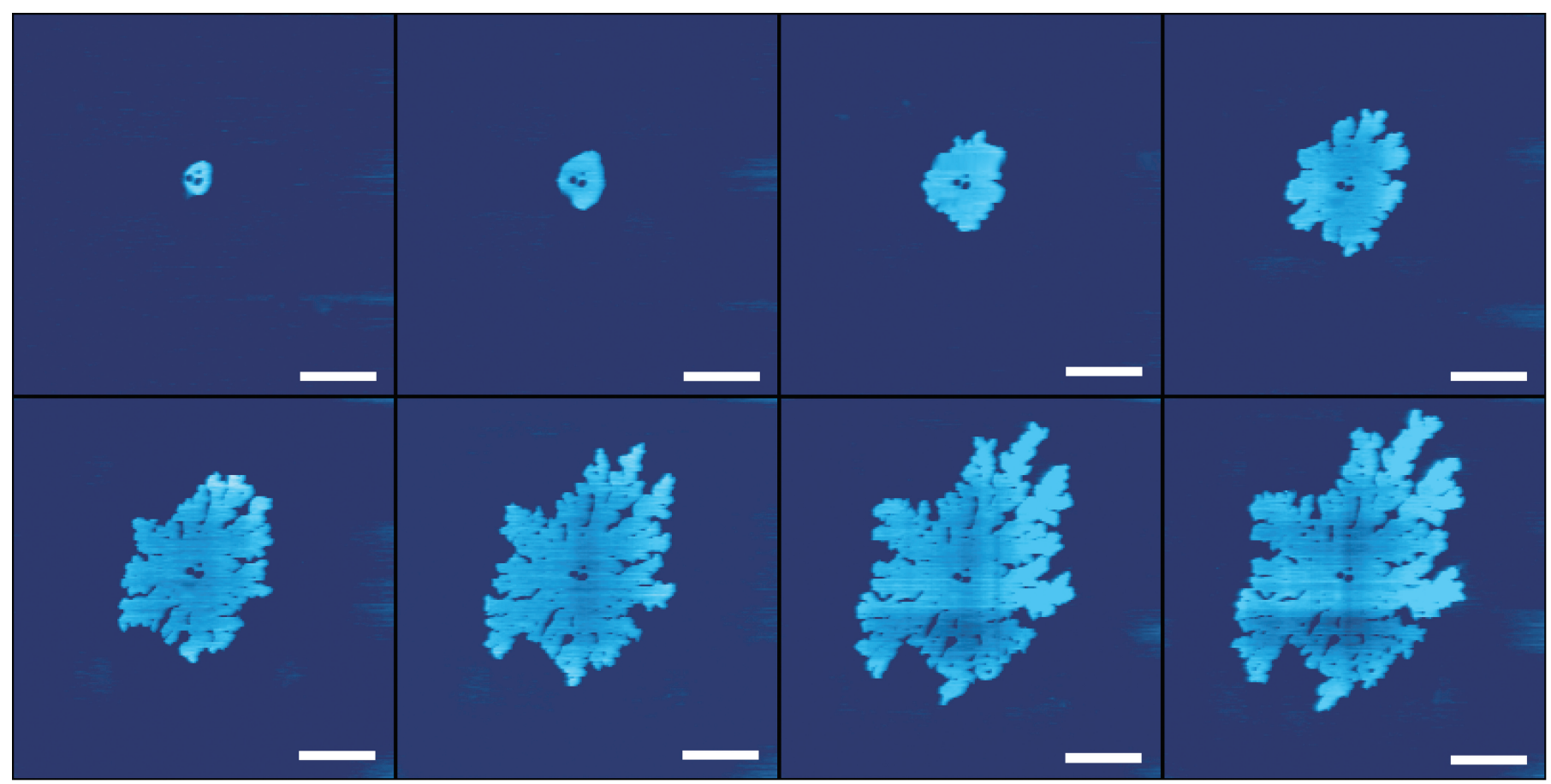

Figure 2: Formation of ice crystals. The formation of an ice crystal at the nano scale was investigated by AAC mode imaging. The scan size was $5 \mu m \times 5 \mu m$, and the scan rate was 0.4 frames/s. In this figure every fourth frame is shown. It can be seen that ice grows from a small nucleus into a structure with many branches.

All imaging was done in acoustic AC (AAC) mode, where the AFM probe is excited to oscillate and the feedback keeps the response amplitude of the cantilever constant. For phase imaging, the phase of the cantilever response was recorded in addition to the sample height.

Sample preparation. PDES was deposited on a Si wafer by rubbing at room temperature. This procedure was used to orient the polymer. For melting this sample, the temperature was varied using a heating stage.

For the ice crystallization study, a freshly cleaved mica sheet was mounted on a cooling sample stage. To find the best

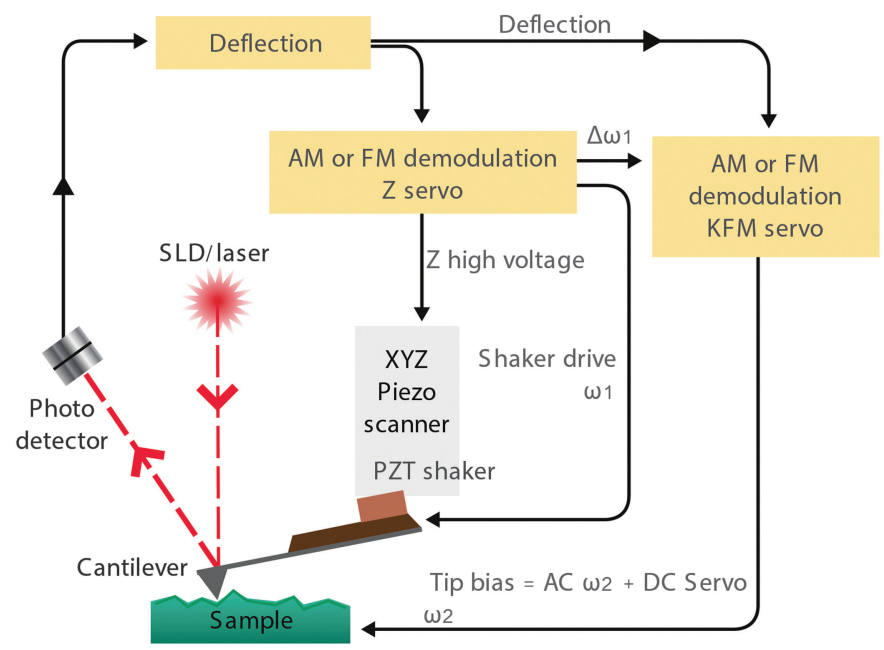

Figure 3: Sketch of single-pass KFM implementation. Two servo-loops are employed for simultaneous detection of mechanical and electrostatic tip-sample interactions. Topography profiling is arranged at the probe resonant frequency $\omega_{1}$ with the $Z$ servo. The KFM servo operates at $\omega_{2}$, which typically is set to be much less than $\omega_{1}$ and is based on the deflection or phase signal, respectively, in AM and FM approaches. condition for studying ice crystal formation, the environmental control capabilities of the instrument were used to generate environments with different humidity levels.

Single-pass Kelvin force microscopy (KFM). The basic principle of KFM is based on the measurement of electrostatic forces between tip and sample. For a DC bias $\left(V_{D C}\right)$ with a small modulation signal $\left(V_{A C} \sin \omega_{2} t\right)$ applied, the induced capacitive force is:

$$
\begin{gathered}
F(z)=-\frac{1}{2} \frac{\partial C}{\partial z}\left[\left(V_{D C}-\phi\right)^{2}+\frac{1}{2} V_{A C}^{2}\right]- \\
\frac{\partial C}{\partial z}\left(V_{D C}-\phi\right) V_{A C} \sin \omega_{2} t+\frac{1}{4} \frac{\partial C}{\partial z} V_{A C}^{2} \cos 2 \omega_{2} t
\end{gathered}
$$

Where $\phi$ is the contact potential difference (CPD) between tip and sample, $C$ is the capacitance and $z$ is the height. It can be seen that the first $\omega_{2}$ dependent term becomes zero if $V_{D C}=\phi$. Therefore, the surface potential can be measured by nullifying the $\omega_{2}$ component. The $\cos 2 \omega_{2} \mathrm{t}$ term is proportional to $\partial \mathrm{C} / \partial \mathrm{z}$, thus mapping the amplitude of the cantilever deflection signal at $2 \omega_{2}$ yields an image of these variations, which can be attributed to spatial variations of local dielectric properties. For KFM experiments EFM probes from NanoWorld (Neuchâtel, Switzerland) were used. The acoustic excitation frequency $\omega_{1}$ was set to $78 \mathrm{kHz}$, which is just below the resonance frequency of the cantilever, and the electrical frequency $\omega_{2}$ was set to $15 \mathrm{kHz}$, well outside the bandwidth of the acoustic amplitude detection, to not interfere with topography profiling.

Instrument improvements. In the past operating an AFM required considerable experience because there are many operational modes, requiring specific setups for the instrument. In order to reduce this experience barrier new software was developed. The graphical user interface (GUI) was designed to guide novice users through the necessary steps to acquire data. 

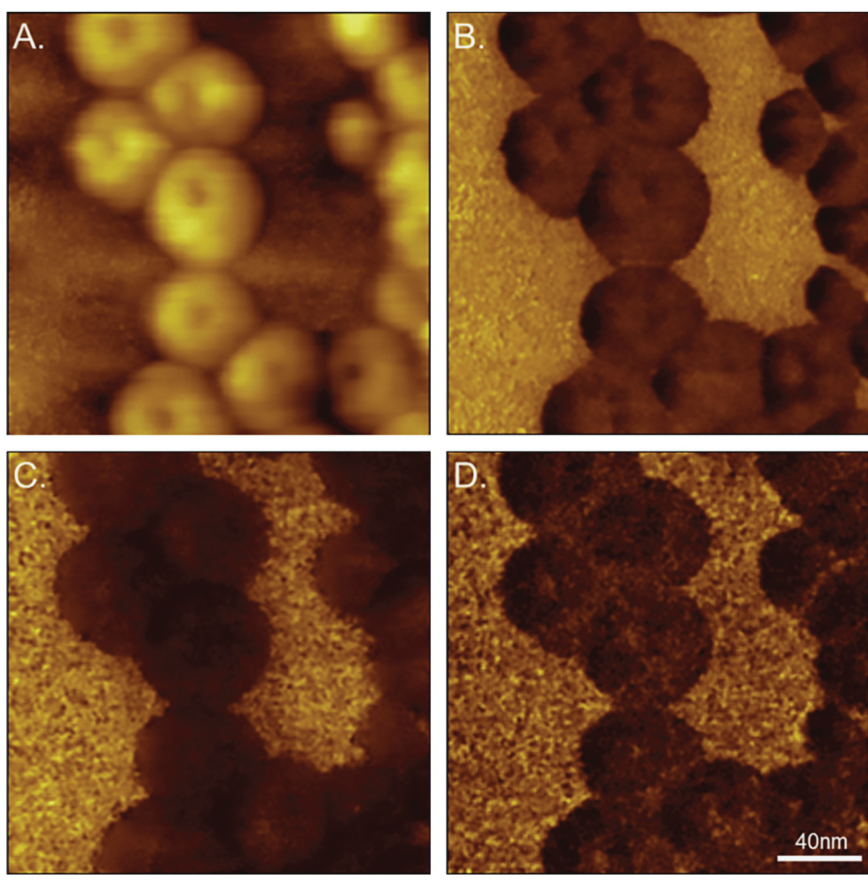

Figure 4: KFM experiments of $\mathrm{CF}_{3}\left(\mathrm{CF}_{2}\right)_{14}\left(\mathrm{CH}_{2}\right)_{20} \mathrm{CH}_{3}$. The topography(A) of individual molecules shows a toroidal structure. The center hole can be observed in the topography (A), phase (B), surface potential (C), and dC/dz (D) channel. This shows the high spatial resolution achievable by KFM.

This was achieved by implementing a workflow-based approach. The GUI guides the user through the steps "LOAD," "PREPARE," "APPROACH," and "MEASURE." Most settings are either automatically obtained or have meaningful default values. Furthermore, only values requiring user input or that are commonly changed are shown. This results in a clear path to obtain experimental data, yielding a higher throughput.

To image a sample surface, a feedback loop keeps the tip deflection constant. In the past the parameters of this feedback loop were set by the operator. In order to obtain good AFM images, it was necessary for the operator to have a lot of experience. The new 9500 AFM implements an automated feedback parameter calculation called "AutoDrive." For this, an accurate and robust AFM system model was developed, which is used to calculate optimized feedback parameters using control theory [5]. Now even novice users are able to acquire useful AFM images.

A major drawback of conventional AFM imaging was its low speed. Typically, it took several minutes to obtain an image, which made it inconvenient to use. Further, many dynamic processes were impossible to investigate. In order to improve this situation, the mechanical tip positioning system was built using a stiff flexure based design. This resulted in an increased mechanical bandwidth, allowing the tip to move faster across the sample surface. In order to get accurate sample tracking at these faster tip movements, a faster controller was required, and the digital high-bandwidth field programmable gate array controller was developed to meet this need. It allows for suitable force control even at high speed, necessary for good sample tracking, while not interfering with the sample. An additional increase in imaging speed was achieved by developing higherorder control techniques to compensate for mechanical resonances [6].

\section{Results}

Polymer phase transition. PDES is a polymer that exists in different states: it is crystalline below $7^{\circ} \mathrm{C}$ and amorphous at temperatures higher than $47^{\circ} \mathrm{C}$. In the intermediate temperature range it is in a partially crystalline state. In order to understand the phase transition on the nanoscale, AFM experiments were performed. For this we prepared a PDES sample on a Si wafer and used the temperature control capability of the 9500AFM to heat the sample from room temperature to $48^{\circ} \mathrm{C}$ while we performed AFM imaging. In order to not interfere with this soft sample, we used the gentle acoustic AC (AAC) mode. In this mode we excite the cantilever by means of a piezo shaker to oscillate close to its resonance frequency, and the feedback loop keeps the oscillation amplitude constant. Because the cantilever touches the sample only at the very end of its downward swing, the forces applied to the sample are small, and even soft samples are not altered. Figure 1 shows such AFM images acquired at a rate of 0.1 frames/s. As expected, at room temperature a partially crystalline structure can be seen. During heating the crystalline features melt and the material becomes amorphous. Subsequent cooling resulted in formation of crystalline structures again.

Ice crystallization. Another important phase transition process is the formation of water ice crystals; in particular, the crystallization of ice from gas phase. Understanding this process could be used to develop materials that prevent this formation, especially on airplanes where the formation of ice is unwanted. In order to understand this process, we used AFM imaging for investigation. We placed a sheet of mica on a temperaturecontrolled stage and cooled the stage below the freezing point of water. We also used the environmental chamber of the system to provide a humid environment. Experiments showed that a $27 \%$ humidity level resulted in good conditions for forming ice crystals. The process of ice formation was observed by AAC mode imaging. Images were recorded at a rate of 0.4 frames/s to capture the crystallization process. Figure 2 shows every fourth image of this process. It can be seen that the crystal forms from a nucleus into a structure with many branches. In addition, a video was acquired of this process [7].

Surface potential measurement. Surface potential at the nanoscale can be measured using KFM. This direct and quantitative measurement of surface potential at high spatial resolution results in important information such as surface charging, molecular dipole orientation in organic thin films, and band bending and dopant concentration in semiconductor materials. By using the single-pass KFM technique, topography and surface potential can be measured simultaneously. This is achieved by applying a dual-frequency excitation to the AFM probe. One excitation signal $\left(\omega_{1}\right)$ is used for modulating the AFM tip for AAC imaging, while the second one $\left(\omega_{2}\right)$ is applied to modulate the electrostatic force. A feedback loop is used nullify the deflection amplitude at $\omega_{2}$ by adding a DC bias (Figure 3). 
Then the surface potential is equal to this applied DC bias (see Materials and Methods) [8]. Because $\omega_{1}$ and $\omega_{2}$ are different, both control loops are active simultaneously resulting in topography and surface potential images acquired at the same time.

Fluoroalkane molecules $\left(\mathrm{CF}_{3}\left(\mathrm{CF}_{2}\right)_{\mathrm{n}}\left(\mathrm{CH}_{2}\right)_{\mathrm{m}} \mathrm{CH}_{3}\right)$ form selfassembled structures on a silicon substrate. Figure 4 shows that the $\mathrm{CF}_{3}\left(\mathrm{CF}_{2}\right)_{14}\left(\mathrm{CH}_{2}\right)_{20} \mathrm{CH}_{3}$ molecule forms toroids. This molecule has a dipole of $3.1 \mathrm{D}$ oriented along the chain at the central $-\mathrm{CH}_{2}-\mathrm{CF}_{2}$ - junction. Macroscopic studies showed a surface potential of $-0.8 \mathrm{~V}$, while KFM measurements on the nanoscale resulted in $-0.75 \mathrm{~V}$ (Figure 4). The $\mathrm{dC} / \mathrm{dz}$ channel shows even contrast associated to local dielectrical properties of this material.

\section{Conclusion}

AFM can image samples at sub-nm resolution under various environmental conditions, including vacuum, ambient, and liquid. In the past, operating these instruments required considerable experience. Furthermore, the speed of acquisition was low. The newest generation of AFM is a game changer. A new software interface guides the user through the acquisition process and automates most of the settings. In addition, the acquisition speed has been increased dramatically, allowing the AFM, for the first time, to be used to capture dynamical processes. In this article we investigated the processes of melting a polymer and of ice formation using temperature and environmental control. In addition, we showed some electrical characterization results.

\section{References}

[1] G Binnig et al., Phys Rev Lett 56 (1986) 930-33.

[2] FJ Giessibl, Science 267 (1995) 68-71.

[3] C Rankl et al., P Natl Acad Sci 105 (2008) 17778-83.

[4] Keysight Technologies Inc., "N9417S-N9613A 9500 Atomic Force Microscope (AFM)." http://www.keysight.com/en/ pd-2569379/9500-atomic-force-microscope-afm?nid=33986.1132645\&cc=US\&lc=eng (accessed August 12, 2016).

[5] DY Abramovitch et al., "Automatic Generation of PID Parameters for a Scanning Probe Microscope". US Patent 7987002, 26072011.

[6] CR Moon. "Atomic Force Microscopy Controller and Method”. US Patent 9.229,027 B2, 5 Jan 2016.

[7] Keysight Technologies Inc., "Ice crystallization on mica," published September 29, 2015. https://www.youtube.com/ watch?v=CELs_5usWk4 (accessed 20 August 2016).

[8] S Maganov and J Alexander, Compositional Mapping of Materials with Single-Pass Kelvin Force Microscopy. Keysight Technologies Inc., Santa Rosa, CA, 2014.

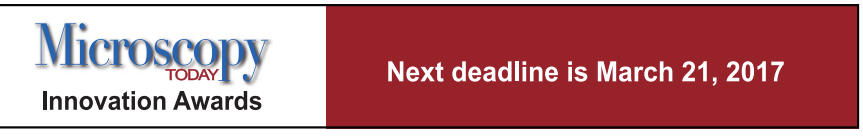

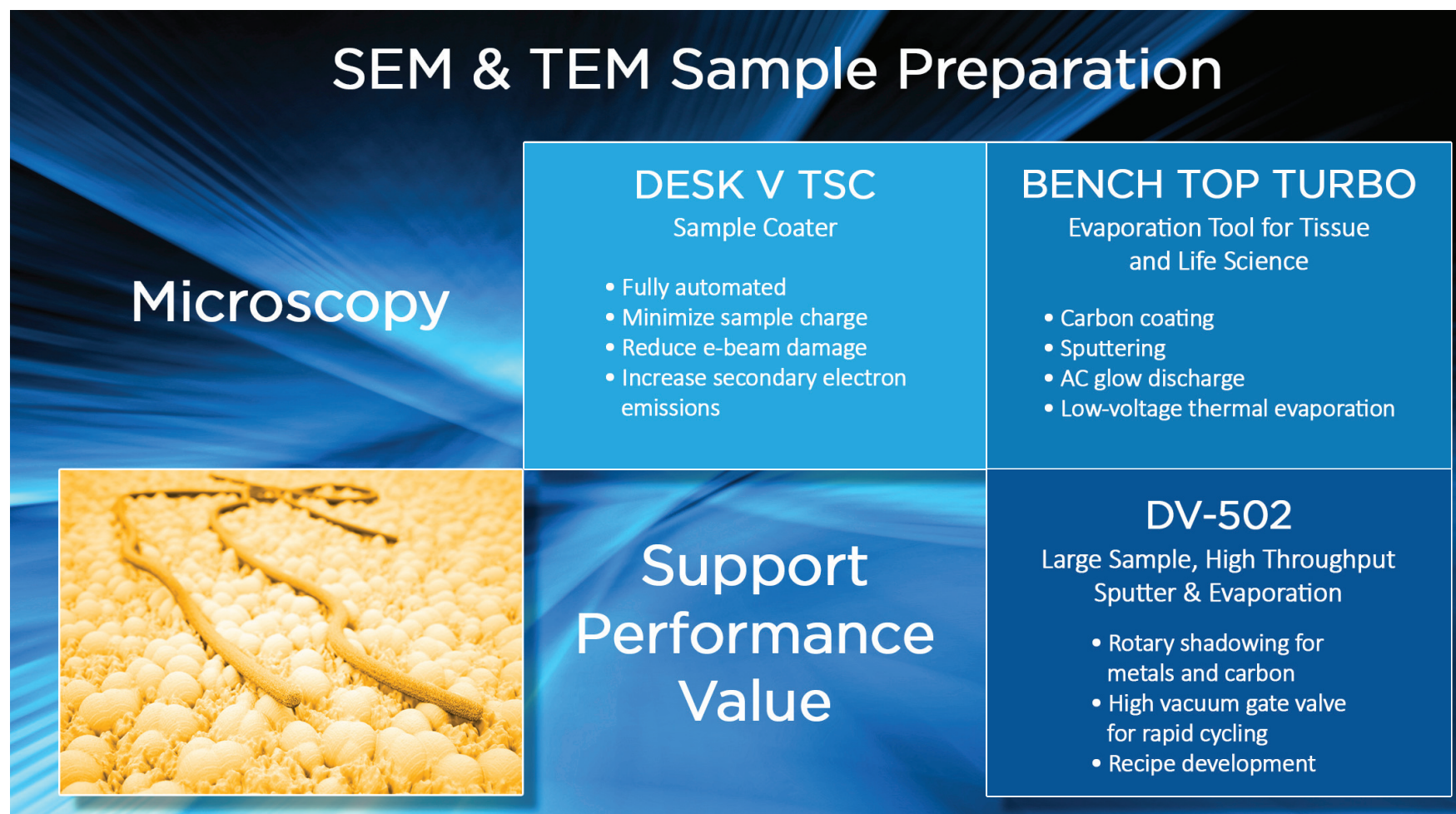




\section{STELLAP RESபLTS}

AT $⿴$ THE ATOMIC SCALE

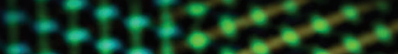
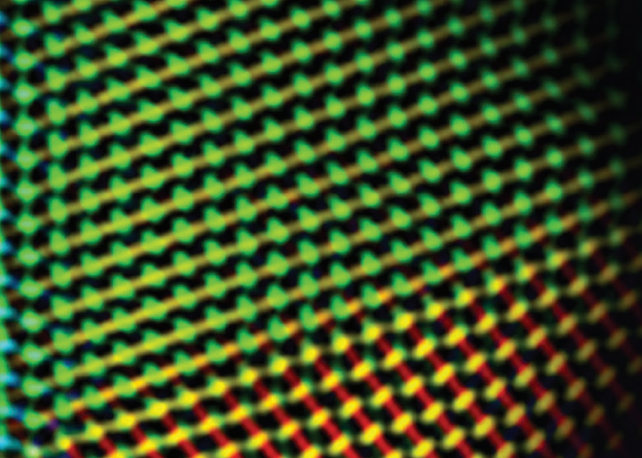

\section{ARM-200F • ARM-300F Atomic Resolution TEM}

- Highest resolution commercially-available S/TEM

- $200 \mathrm{kV}$ and $300 \mathrm{kV}$ models

- $\mathrm{C}_{\mathrm{s}}$ correctors - innovative options

- Cold FEG

- Superior SDD technology

- Ultimate stability
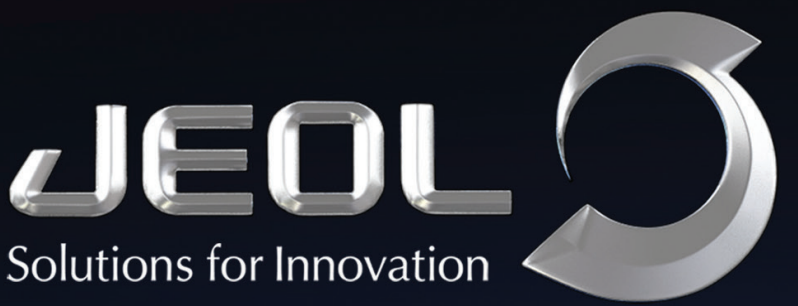

Solutions for Innovation

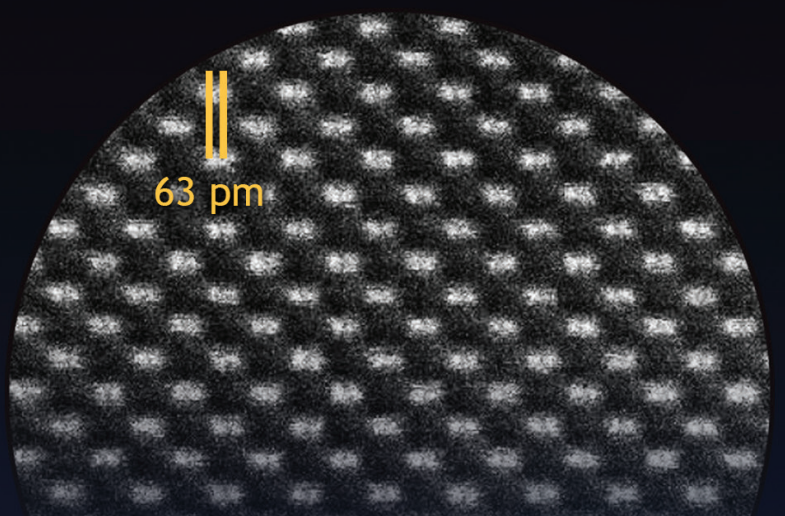

\section{Learn more at jeolusa.com/atomicresolutionTEM}

A leader never stops innovating

www.jeolusa.com • salesinfo@jeol.com 